\title{
FUNGOS MICORRÍZICOS NO CRESCIMENTO E NUTRI- ÇÃO DE REBENTOS ORIUNDOS DE COROA DE ABACAXI ${ }^{1}$
}

\author{
PAULO CESAR DOS SANTOS², MARTA SIMONE MENDONÇA FREITAS ${ }^{3}$, SÍLVIO DE JESUS \\ FREITAS ${ }^{4}$, MÍRIAN PEIXOTO SOARES DA SILVA ${ }^{5}$, SÁVIO DA SILVA BERILLI ${ }^{6}$
}

RESUMO - A produção nacional de mudas de abacaxi tem sido caracterizada pela baixa oferta de material nos padrões recomendados. O uso da técnica de eliminação da dominância, apical aliado à utilização de fungos micorrízicos arbusculares (FMAs), pode proporcionar aumento na produtividade do viveiro e menor tempo de produção das mudas. Nesse sentido, o objetivo deste estudo foi avaliar a ação de FMAs no crescimento de rebentos de abacaxi originários através da técnica de eliminação do meristema apical, de coroas também inoculadas com estes FMAs. Utilizou-se o delineamento em blocos casualizados, num fatorial $2 \times 3$, com duas cultivares de abacaxi ('Smooth Cayenne' e 'Pérola') e três tratamentos microbiológicos (sem inoculação, inoculação com Glomus etunicatum e inoculação com uma mistura dos fungos Glomus clarum e Gigaspora margarita), com quatro repetições. Conclui-se que a inoculação com FMAs no crescimento das mudas não proporciona redução na fase de enviveiramento. A inoculação de FMAs na cultivar Pérola mostrou-se não benéfica, e o tratamento microbiológico com a mistura apresentou-se como parasita, reduzindo o teor nutricional de P e K, não sendo indicado para esta cultivar. Para o 'Smooth Cayenne', a inoculação com FMAs proporcionou incremento de $\mathrm{P}$ nas mudas.

Termos para indexação: Ananas comosus var. comosus, fusariose, abacaxizeiro, mudas, propagação.

\section{MYCORRHIZAL FUNGI ON GROWTH AND NUTRITION OF SPROUTS FROM PINEAPPLE CROWN}

\begin{abstract}
The use of arbuscular mycorrhizal fungi (AMF) can be an alternative to improve the production of seedlings, since they can shorten the time of seedlings formation in several fruits. The objective of this study was to evaluate the effect of AMF on the growth of pineapple shoots from the technique of removing the apical meristem of crowns also inoculated with AMF. The experiment was set up in randomized block design in a $2 \times 3$ factorial, with two pineapple cultivars ('Smooth Cayenne' and 'Pearl') and three microbiological treatments (no inoculation, inoculation with Glomus etunicatum and inoculation with a mixture of the fungi Glomus clarum and Gigaspora margarita, with four replications. The inoculation with AMF, provided no reduction in the duration of the seedlings production. The AMF inoculation on cultivar Pearl showed to be not beneficial, the microbiological treatment with the mixture performed like a parasite, reducing the nutritional content of $\mathrm{P}$ and $\mathrm{K}$ and is not suitable for this cultivar. In the other hand, the 'Smooth Cayenne' inoculation with AMF provided an increase of the P content in the seedlings.
\end{abstract}

Index terms: Ananas comosus var. comosus, fusariosis, pineapple, seedlings, propagation.

\section{INTRODUÇÃO}

A produção nacional de mudas de abacaxi tem sido caracterizada pela baixa oferta de material de qualidade sanitária e da falta de um sistema eficiente de obtenção de mudas. A disponibilização destas no mercado ainda é considerada um desafio, pois o período de permanência no viveiro eleva sobremaneira o custo de produção das mudas, o que acaba muitas vezes inviabilizando esta atividade por parte dos viveiristas.

O aprimoramento e o desenvolvimento de novas técnicas de propagação, que tenham maior eficácia, rapidez e simplicidade na execução, tornam-se fundamentais (COELHO et al., 2007) para aquisição de mudas de qualidade e com menores custos.

No intuito de atender a estes critérios, Coelho et al. (2007) e Santos (2009) testaram uma técnica

\footnotetext{
${ }^{1}$ Trabalho Sinfruit 119 - Simpósio Internacional de Fruticultura - Avanços na Fruticultura (17 a 21 Outubro) ${ }^{2}$ Mestrando em Produção Vegetal, UENF/CCTA/LFIT, 28013-603, Campos-RJ. E-mail: pcsantos18@hotmail.com ${ }^{3}$ DSc em Produção Vegetal, Pos-doutoranda, UENF/CCTA, 28013-602, Campos-RJ. E-mail: msimone@uenf.br ${ }^{4}$ DSc em Produção Vegetal, Pos-doutorando, UENF/CCTA, 28013-603, Campos-RJ. E-mail: freitassj@yahoo.com.br ${ }^{5}$ Doutoranda em Produção Vegetal, UENF/CCTA/LFIT, 28013-603, Campos-RJ. E-mail: mirianpsoares@gmail.com ${ }^{6}$ DSc em Produção Vegetal, UENF/CCTA/LFIT, 28013-603, Campos-RJ. E-mail: berilli@gmail.com
} 
de propagação vegetativa do abacaxi, que consiste na destruição do meristema apical da coroa, visando à quebra da dominância apical. Essa interferência mecânica provoca o desenvolvimento das gemas laterais, as quais originam os rebentos.

O uso desta técnica, aliada à utilização de fungos micorrízicos arbusculares (FMAs), pode proporcionar aumento na produtividade do viveiro e menor tempo de produção das mudas, visto que os FMAs, ao estabelecerem uma associação simbiótica com a muda, passam a explorar um volume maior de substrato, favorecendo a absorção de nutrientes. Esse processo reflete diretamente na qualidade da muda e em sua capacidade de tolerar estresses ambientais no pós-plantio.

Santos (2009) verificou a viabilidade do uso de FMAs em coroas de abacaxi, na produção de rebentos. Entretanto, ainda não é conhecido o efeito desta associação no crescimento desses rebentos.

Neste sentido, o objetivo deste estudo foi avaliar a ação de FMAs (Glomus etunicatum e mistura dos fungos Glomus clarum e Gigaspora margarita) no crescimento de rebentos de duas cultivares de abacaxi (Smooth Cayenne e Pérola) originários através da técnica de eliminação do meristema apical, de coroas também inoculadas com estes FMAs.

\section{MATERIAL E MÉTODOS}

O experimento foi conduzido em casa de vegetação no Câmpus da Universidade Estadual do Norte Fluminense Darcy Ribeiro - UENF, em Campos dos Goytacazes - RJ, situada a $21^{\circ} 48^{\prime}$ de latitude sul, $41^{\circ} 20^{\prime}$ de longitude $\mathrm{W}$, altitude de $11 \mathrm{~m}$, no período de agosto a dezembro de 2009 , totalizando 150 dias.

O delineamento experimental adotado foi o de blocos ao acaso (DBC), em sistema fatorial $2 \mathrm{X} 3$, sendo a inoculação de mudas (com aproximadamente 10 $\mathrm{cm})$ do tipo rebento, de duas cultivares de abacaxi ('Smooth Cayenne' e 'Pérola') e três tratamentos microbiológicos (sem inoculação, inoculação com Glomus etunicatum e inoculação com uma mistura dos fungos Glomus clarum e Gigaspora margarita), com quatro repetições para cada tratamento.

Os inóculos foram adquiridos da coleção do Setor de Microbiologia do Laboratório de Solos da UENF. A multiplicação dos fungos ocorreu em casa de vegetação, utilizando-se como substrato de uma mistura de solo e areia na proporção de 1:1 (v/v), respectivamente, autoclavados por duas vezes, a uma temperatura de $121^{\mathrm{oC}}$ por $1 \mathrm{~h}$. Utilizaram-se vasos com capacidade de $3 \mathrm{dm} 3$, nos quais foram adicionados o substrato e $50 \mathrm{~g}$ de inóculo (mistura de solo contendo esporos, hifas e raízes colonizadas) das espécies de fungos a serem estudadas. Em seguida, 10 sementes de milho (Zea mays) foram semeadas em cada vaso, sendo que, antes do plantio, as sementes foram desinfestadas com solução de $0,5 \%$ de hipoclorito de sódio, durante 10 minutos, e depois lavadas com água desionizada. Quatro meses após o plantio do milho, a parte aérea foi cortada e, 30 dias após o corte, a mistura do solo contendo esporos, hifas e raízes finas foi utilizada como inóculo nos tratamentos do experimento.

O substrato utilizado para o transplantio dos rebentos foi uma mistura de terra de superfície, areia lavada e de substrato Plantmax ${ }^{\circledR}$ hortaliça nas proporções 4:2:1 (v/v), respectivamente. Posteriormente, este substrato foi esterilizado em autoclave por duas vezes, a uma temperatura de $121^{\circ} \mathrm{C}$ por $1 \mathrm{~h}$, com a finalidade de eliminação dos FMAs nativos. Após a autoclavagem, o substrato apresentou as características químicas da Tabela 1.

Os rebentos foram obtidos de brotações de gemas de coroas de abacaxi, nas quais foi quebrada a dominância apical manualmente, com auxílio de um alicate com ponta fina e uma chave de fenda (COELHO et al., 2007; SANTOS, 2009).

$\mathrm{O}$ experimento foi conduzido em jardineiras $(75$ X 20 X $20 \mathrm{~cm})$, sendo adicionadosk o substrato e $100 \mathrm{~g}$ do inóculo (mistura do solo contendo esporos, hifas e raízes finas cortadas) de 2 a $3 \mathrm{~cm}$ abaixo da superfície do substrato, de acordo com os tratamentos.

Após o transplantio dos rebentos, foram monitorados o comprimento e o número de folhas, em quatro épocas (45; 90; 120 e 150 dias). Para o comprimento, as mudas tiveram suas folhas agrupadas para cima, sendo medido da base até a extremidade da folha maior.

Ao final do experimento, as mudas de abacaxi foram coletadas e levadas ao laboratório para a avaliação do peso seco da parte aérea (obtidas após a secagem em estufa sob ventilação forçada de ar a $70^{\circ} \mathrm{C}$ até atingir o peso constante).

Depois de secas, as amostras foram moídas em moinho tipo Wiley com peneira de 20 mesh e armazenadas em frascos hermeticamente fechados. Para a determinação dos nutrientes do material moído, foram pesadas duas amostras de cada tratamento para se proceder, respectivamente, às digestões sulfúricas (determinação dos teores de N) e nitro-perclórica (determinação dos teores de $\mathrm{P}, \mathrm{K}, \mathrm{Ca}, \mathrm{Mg}$ e $\mathrm{S}$ ).

$\mathrm{O} N$ orgânico foi dosado pelo método de Nessler (JACKSON, 1965). O K foi dosado por espectrofotometria de emissão atômica; o P determinado, colorimetricamente, pelo método do molibdato; o $\mathrm{Ca}$ e $\mathrm{Mg}$ por espectrometria de absorção atômica 
(MALAVOLTA et al., 1997).

Para a avaliação da infecção micorrízica, as raízes foram removidas dos vasos, lavadas com água corrente. As raízes finas foram armazenadas em álcool etílico a 50\% para proceder à avaliação da porcentagem de colonização radicular. Para isto, as raízes foram coloridas segundo Grace e Stribley (1991), com as seguintes adaptações: clareamento das raízes por cinco minutos a $80^{\circ} \mathrm{C}$ com $\mathrm{KOH} 2,5 \%$, e posterior coloração por $20 \mathrm{~min}$ a $80^{\circ} \mathrm{C}$ com azul de metil. Em seguida, as raízes foram levadas ao microscópio estereoscópico com aumento de $20 \mathrm{X}$ para determinar o grau de infecção.

Os dados foram submetidos a análises de variância pelo teste $\mathrm{F}$, as variáveis quantitativas foram submetidas à análise de regressão, e as qualitativas ao teste de Tukey, a $5 \%$ de probabilidade de erro.

\section{RESULTADOS E DISCUSSÃO}

$\mathrm{Na}$ análise de colonização do sistema radicular, verificou-se que todos os tratamentos microbiológicos (exceto o controle) foram capazes de se associar às raízes do abacaxizeiro. $\mathrm{O}$ inóculo misto foi capaz de colonizar ambas as cultivares de forma igual. Diferentemente foi verificado para o inóculo G. etunicatum, este apresentou maior índice de colonização na cultivar Pérola, quando comparado ao 'Smooth Cayenne'. Nas cultivares avaliadas, sempre o inóculo misto colonizou mais que o G. etunicatum (Tabela 2).

A introdução FMAs tem um grande potencial nos programas de produção de mudas, pois permite abreviar o tempo de formação da muda de várias fruteiras, como bananeira (LINS et al., 2003), cajueiro (WEBER et al., 2004), maracujazeiro-doce (ANJOS et al., 2010) e graviola (SAMARÃO et al., 2011). O tempo requerido para produção de mudas de abacaxi é crítico, pois apresenta crescimento lento, aumentando o período de permanência no viveiro.

Segundo Matos et al. (2010), uma muda de abacaxi pode ser considerada adequada para o plantio definitivo quando atingir a altura mínima de $25 \mathrm{~cm}$. Este valor foi alcançado aos $105 ; 110 ; 119 ; 120 ; 124$ e 125 dias para os tratamentos: 'Pérola' sem inoculação, 'Pérola' inoculado com a mistura, 'Smooth Cayenne' inoculado com o G. etunicatum, 'Pérola' inoculado com o G. etunicatum, 'Smooth Cayenne' sem inoculação e 'Smooth Cayenne' inoculado com a mistura, respectivamente (Figura 1).

A inoculação com FMAs causou diminuição no número de folhas para ambas as cultivares (Figura 2). Resultados diferentes foram observados por Gutierrez-Oliva et al. (2009), em que a inoculação com Glomus claroideum em mudas de abacaxizeiro micropropagadas promoveu maior número de folhas e peso seco.

Esses resultados divergentes são explicados devido à eficiência micorrízica estar relacionada com a quantidade de micélio externo formado no solo. Apesar de certos fungos possuírem capacidade de colonizar o hospedeiro, a proporção de hifas externas formadas (estruturas que permitem a maior absorção de nutrientes) varia muito entre as espécies de FMAs (MARSCHNER; DELL, 1994). A eficiência da colonização micorrízica é dependente do genótipo da planta, da espécie do fungo e do ambiente. Essa colonização pode proporcionar baixo crescimento vegetal ou até chegar a atingir uma condição de parasitismo, quando o balanço energético se torna desfavorável à planta. Stancato et al. (2010) verificaram que a micorrização de plântulas micropropagadas de antúrio, tanto na fase de aclimatização quanto de obtenção de mudas, não promoveu crescimento.

Foi verificado que os tratamentos microbiológicos não influenciam no peso seco da parte aérea das mudas na cultivar Smooth Cayenne. Para a 'Pérola', os tratamentos com FMAs promoveram menor peso seco (Tabela 3). Entretanto, Jaizme-Vega e Azcón (1991) aumentaram a produção de biomassa de mudas micropropagadas de abacaxizeiro 142\% e $148 \%$ com a inoculação de $G$. fasciculatum e $G$. mosseae, respectivamente.

Não houve interação entre os tratamentos para as variáveis: peso seco de raiz das mudas e os teores nutricionais $(\mathrm{N}, \mathrm{Ca}$ e $\mathrm{Mg}$ ) na parte aérea das mudas. Entre as cultivares, o 'Smooth Cayenne' produziu $139 \%$ mais peso seco de raiz do que o 'Pérola'. Quanto aos teores nutricionais entre as cultivares, foram verificados valores superiores de $\mathrm{N}$ e $\mathrm{Ca}$ na cultivar Smooth Cayenne e Mg para a 'Pérola' (Tabela 4).

Os tratamentos microbiológicos que continham inoculação com fungos micorrízicos reduziram o peso seco da raiz em torno de $61 \%$ (G. etunicatum) e $41 \%$ (mistura dos fungos) em relação ao sem fungo (Tabela 5).

Constatou-se efeito do tratamento microbiológico sobre a nutrição das mudas, a inoculação com a mistura e o G. etunicatum proporcionaram incrementos significativos de $\mathrm{N}$ e $\mathrm{Mg}$, em relação ao tratamento sem inoculação. Para o $\mathrm{Ca}$, a mistura dos fungos promoveu incremento de $5,14 \%$ em relação ao G. etunicatum e $8,32 \%$, em relação ao tratamento sem fungo (Tabela 5). Esse maior aporte de nutrientes deve-se aos FMAs expandirem a zona de absorção da raiz. Marschner e Dell (1994) verificaram que os fungos micorrízicos podem ser responsáveis pela absorção de cerca de $80 \%$ de $\mathrm{P}, 25 \%$ do $\mathrm{N}$ e $10 \%$ de $\mathrm{K}$. 
Os tratamentos microbiológicos influenciaram nos teores de $\mathrm{P}$ de forma diferente entre as cultivares. A inoculação com os FMAs promoveram maior absorção de P para cultivar Smooth Cayenne. No 'Pérola', quando inoculado com a mistura, o teor de P foi menor em relação ao sem fungo. No entanto, estes dois não diferiram do tratamento com $G$. etunicatum. Os teores de $\mathrm{P}$ foram maiores no 'Pérola' quando aplicados os tratamentos com G. etunicatum e sem fungo (Tabela 6).

Trindade et al. (2001) observaram, em mudas de mamoeiro do grupo solo e formosa, que a inoculação com G. margarita proporcionou maiores teores e conteúdos de $\mathrm{P}$ na parte aérea, quando comparados ao tratamento sem inoculação.

Para o K, os tratamentos microbiológicos não diferiram para a cultivar Smooth Cayenne. A inoculação com a mistura no 'Pérola' diminuiu em $11,98 \%$ o teor de K em relação ao G. etunicatum e $10,31 \%$ quando comparado ao tratamento sem fungo. A cultivar Smooth Cayenne obteve maior teor de K do que a 'Pérola' (Tabela 7).

Freitas et al. (2006) observaram que a inoculação de plantas de menta com G. clarum e $G$. margarita elevaram os conteúdos de $\mathrm{K}$ na parte aérea em 139 ou $142 \%$, respectivamente, quando avaliadas em comparação com os teores observados em plantas não inoculadas.

TABELA 1- Análise química do substrato após autoclavagem por duas vezes, a uma temperatura de $121^{\circ} \mathrm{C}$ por 1 hora.

\begin{tabular}{|c|c|c|c|c|c|c|c|c|c|c|c|c|c|c|}
\hline & $\mathrm{K}$ & $\mathrm{Ca}$ & $\mathrm{Mg}$ & $\mathrm{Al}$ & $\mathrm{H}+\mathrm{Al}$ & SB & \multirow{2}{*}{$\begin{array}{c}\mathrm{V} \\
(\%) \\
\end{array}$} & $P$ & $\mathrm{Fe}$ & $\mathrm{Cu}$ & $\mathrm{Zn}$ & $\mathrm{Mn}$ & ${\mathrm{S}-\mathrm{SO}^{4}}^{4}$ & B \\
\hline \multirow{2}{*}{5,8} & \multicolumn{6}{|c|}{$\mathrm{mmol}_{\mathrm{c}} \mathrm{dm}^{-3}$} & & \multicolumn{7}{|c|}{$\mathrm{mg} \mathrm{dm}^{-3}$} \\
\hline & 1,6 & 29,4 & 14,5 & 0 & 10,9 & 46,5 & 81 & 22 & $\overline{50,8}$ & 0,43 & 1,73 & 82,4 & 110 & 0,34 \\
\hline
\end{tabular}

Matéria orgânica $=35,7 \mathrm{~g} \mathrm{dm}^{-3}$

Análises realizadas pelo Laboratório de Análise de Solos da Fundenor, Campos dos Goytacazes-RJ.

TABELA 2- Colonização dos FMAs em raízes de mudas do tipo rebento do abacaxizeiro 'Smooth Cayenne' e 'Pérola', em função de tratamentos microbiológicos

\begin{tabular}{ccccc}
\hline \multirow{2}{*}{ Cultivar } & \multicolumn{3}{c}{ Tratamento microbiológico } & \multirow{2}{*}{ Média } \\
\cline { 2 - 4 } & Misto & G. etunicatum Sem fungo & \\
\hline 'Smooth' & $100 \mathrm{Aa}$ & $30 \mathrm{Bb}$ & $0 \mathrm{Ca}$ & 43,33 \\
'Pérola' & $95,0 \mathrm{Aa}$ & $80 \mathrm{Ba}$ & $0 \mathrm{Ca}$ & 58,33 \\
\hline Média & 97,5 & 55,0 & 0 & \\
\hline CV $(\%)$ & \multicolumn{5}{c}{15,44} &
\end{tabular}

Médias seguidas de mesma letra maiúsculas nas linhas e minúscula nas colunas não diferem entre si, pelo teste de Tukey (P>0,05). 1: Tratamento com a mistura dos fungos Glomus clarum e Gigaspora margarita.

TABELA 3- Peso seco (g) da parte aérea dos rebentos aos 150 dias após o transplantio

\begin{tabular}{|c|c|c|c|c|}
\hline \multirow{2}{*}{ Cultivar } & \multicolumn{3}{|c|}{ Tratamento microbiológico } & \multirow{2}{*}{ Média } \\
\hline & Misto $^{1}$ & G. etunicatur & Sem Fungo & \\
\hline 'Smooth & $10,44 \mathrm{Aa}$ & $11,79 \mathrm{Aa}$ & $12,38 \mathrm{Aa}$ & 11,53 \\
\hline 'Pérola' & $7,16 \mathrm{Bb}$ & $5,71 \mathrm{Bb}$ & $11,07 \mathrm{Aa}$ & 7,98 \\
\hline Média & 8,8 & 8,75 & 11,73 & \\
\hline $\mathrm{CV}(\%)$ & & 17,00 & & \\
\hline
\end{tabular}

Médias seguidas de mesma letra maiúsculas nas linhas e minúscula nas colunas não diferem entre si, pelo teste de Tukey ( $\mathrm{P}>0,05)$. 1: Tratamento com a mistura dos fungos Glomus clarum e Gigaspora margarita. 
TABELA 4- Peso seco da raiz - PSR (g) e teores dos nutrientes $\left(\mathrm{g} \mathrm{kg}^{-1}\right)$ no peso seco da parte aérea das mudas do tipo rebento do abacaxizeiro 'Smooth Cayenne' e 'Pérola' aos 150 dias após o transplantio.

\begin{tabular}{ccccc}
\hline \multirow{2}{*}{ Cultivar } & \multirow{2}{*}{ PSR } & \multicolumn{3}{c}{ Nutrientes } \\
\cline { 3 - 5 } & & $\mathrm{N}$ & $\mathrm{Ca}$ & $\mathrm{Mg}$ \\
\hline Smooth & $1,15 \mathrm{a}$ & $14,38 \mathrm{a}$ & $10,40 \mathrm{a}$ & $5,93 \mathrm{~b}$ \\
Pérola & $0,48 \mathrm{~b}$ & $12,73 \mathrm{~b}$ & $5,20 \mathrm{~b}$ & $9,10 \mathrm{a}$ \\
\hline Média & 0,81 & 13,56 & 7,80 & 7,51 \\
\hline CV $(\%)$ & 31,68 & 8,64 & 5,75 & 4,89 \\
\hline
\end{tabular}

Médias seguidas de mesma letra nas colunas não diferem entre si pelo teste de Tukey $(\mathrm{P}>0,05)$.

TABELA 5- Peso seco da raiz - PSR ( $\mathrm{g})$ e teores dos nutrientes $\left(\mathrm{g} \mathrm{kg}^{-1}\right)$ no peso seco da parte aérea das mudas do tipo rebento inoculadas com FMAs aos 150 dias após o transplantio.

\begin{tabular}{ccccc}
\hline Tratamento & \multirow{2}{*}{ PSR } & \multicolumn{3}{c}{ Nutrientes } \\
\cline { 3 - 5 } microbiológico & & $\mathrm{N}$ & $\mathrm{Ca}$ & $\mathrm{Mg}$ \\
\hline Sem fungo & $1,05 \mathrm{a}$ & $11,85 \mathrm{~b}$ & $7,49 \mathrm{~b}$ & $6,99 \mathrm{~b}$ \\
G. etunicatum & $0,65 \mathrm{~b}$ & $13,72 \mathrm{a}$ & $7,75 \mathrm{~b}$ & $7,73 \mathrm{a}$ \\
Misto $^{1}$ & $0,74 \mathrm{~b}$ & $15,09 \mathrm{a}$ & $8,17 \mathrm{a}$ & $7,92 \mathrm{a}$ \\
\hline Média & 0,82 & 13,56 & 7,80 & 7,51 \\
\hline $\mathrm{CV}(\%)$ & 31,68 & 8,64 & 5,75 & 4,89 \\
\hline
\end{tabular}

Médias seguidas de mesma letra nas colunas não diferem entre si, pelo teste de Tukey $(\mathrm{P}>0,05)$.

1: Tratamento com a mistura dos fungos Glomus clarum e Gigaspora margarita.

TABELA 6- Teores do nutriente fósforo $\left(\mathrm{g} \mathrm{kg}^{-1}\right)$ no peso seco da parte aérea das mudas do tipo rebento do abacaxizeiro 'Smooth Cayenne' e 'Pérola' aos 150 dias após o transplantio.

\begin{tabular}{lcccc}
\hline \multirow{2}{*}{ Cultivar } & \multicolumn{3}{c}{ Tratamento microbiológico } & \multirow{2}{*}{ Média } \\
\cline { 2 - 4 } & Misto & G.etunicatum & Sem fungo & \\
\hline 'Smooth' & $1,40 \mathrm{Aa}$ & $1,26 \mathrm{Ab}$ & $0,80 \mathrm{Bb}$ & 1,15 \\
'Pérola' & $1,35 \mathrm{Ba}$ & $1,46 \mathrm{ABa}$ & $1,55 \mathrm{Aa}$ & 1,45 \\
\hline Média & 1,38 & 1,36 & 1,17 \\
\hline CV $(\%)$ & \multicolumn{4}{c}{7,33} \\
\hline
\end{tabular}

Médias seguidas de mesma letra maiúsculas nas linhas e minúscula nas colunas não diferem entre si, pelo teste de Tukey (P>0,05)

1: Tratamento com a mistura dos fungos Glomus clarum e Gigaspora margarita.

TABELA 7- Teores do nutriente potássio $\left(\mathrm{g} \mathrm{kg}^{-1}\right)$ no peso seco da parte aérea das mudas do tipo rebento do abacaxizeiro 'Smooth Cayenne' e 'Pérola' aos 150 dias após o transplantio. Médias seguidas de mesma letra maiúsculas nas linhas e minúscula nas colunas não diferem entre si, pelo teste de Tukey $(\mathrm{P}>0,05)$.

\begin{tabular}{ccccc}
\hline \multirow{2}{*}{ Cultivar } & \multicolumn{3}{c}{ Tratamento microbiológico } & \multirow{2}{*}{ Média } \\
\cline { 2 - 4 } & Misto & G.etunicatum & Sem fungo & \\
\hline 'Smooth' & $29,63 \mathrm{Ab}$ & $27,11 \mathrm{Ab}$ & $29,68 \mathrm{Ab}$ & 28,80 \\
'Pérola' & $38,98 \mathrm{Ba}$ & $44,65 \mathrm{Aa}$ & $43,00 \mathrm{Aa}$ & 42,21 \\
\hline Média & 34,30 & 35,88 & 36,34 \\
\hline CV (\%) & 4,53 & \\
\hline
\end{tabular}

1: Tratamento com a mistura dos fungos Glomus clarum e Gigaspora margarita. 


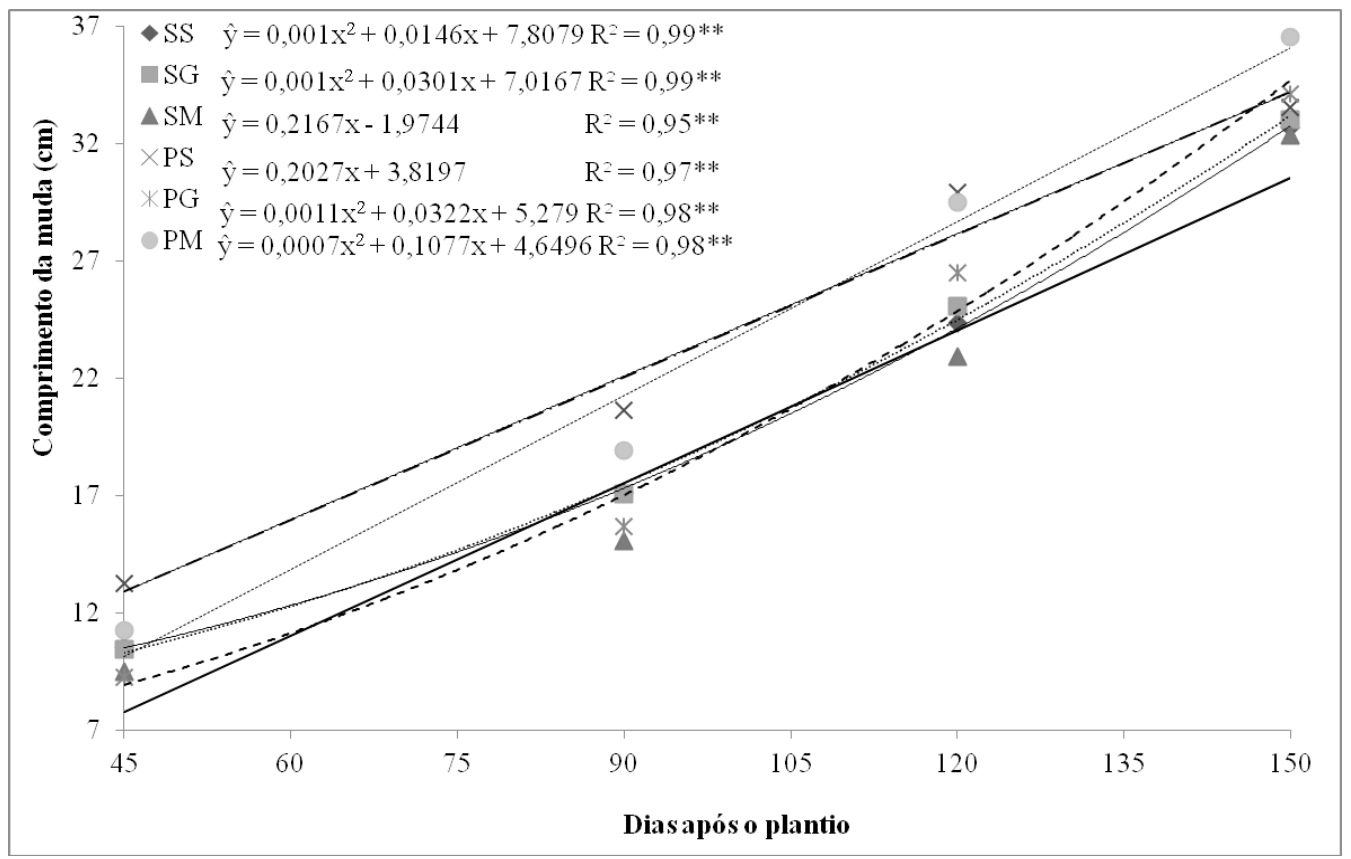

FIGURA 1 - Altura das mudas (cm) do abacaxizeiro 'Pérola' e 'Smooth Cayenne', aplicado três tratamentos microbiológicos em quatro épocas de amostragem. Campos dos Goytacazes - Estado do Rio de Janeiro, 2009.

*Significativo a $1 \% * *$ significativo a $5 \%$

Legenda: SS: 'Smooth Cayenne’ não inoculado, SG: 'Smooth Cayenne’ inoculado com G. etunicatum, SM: 'Smooth Cayenne’ inoculado com mistura dos fungos Glomus clarum e Gigaspora margarita, PS: 'Pérola' não inoculado, PG: 'Pérola' inoculado com G. etunicatum, PM: 'Pérola' inoculado com mistura dos fungos Glomus clarum e Gigaspora margarita.

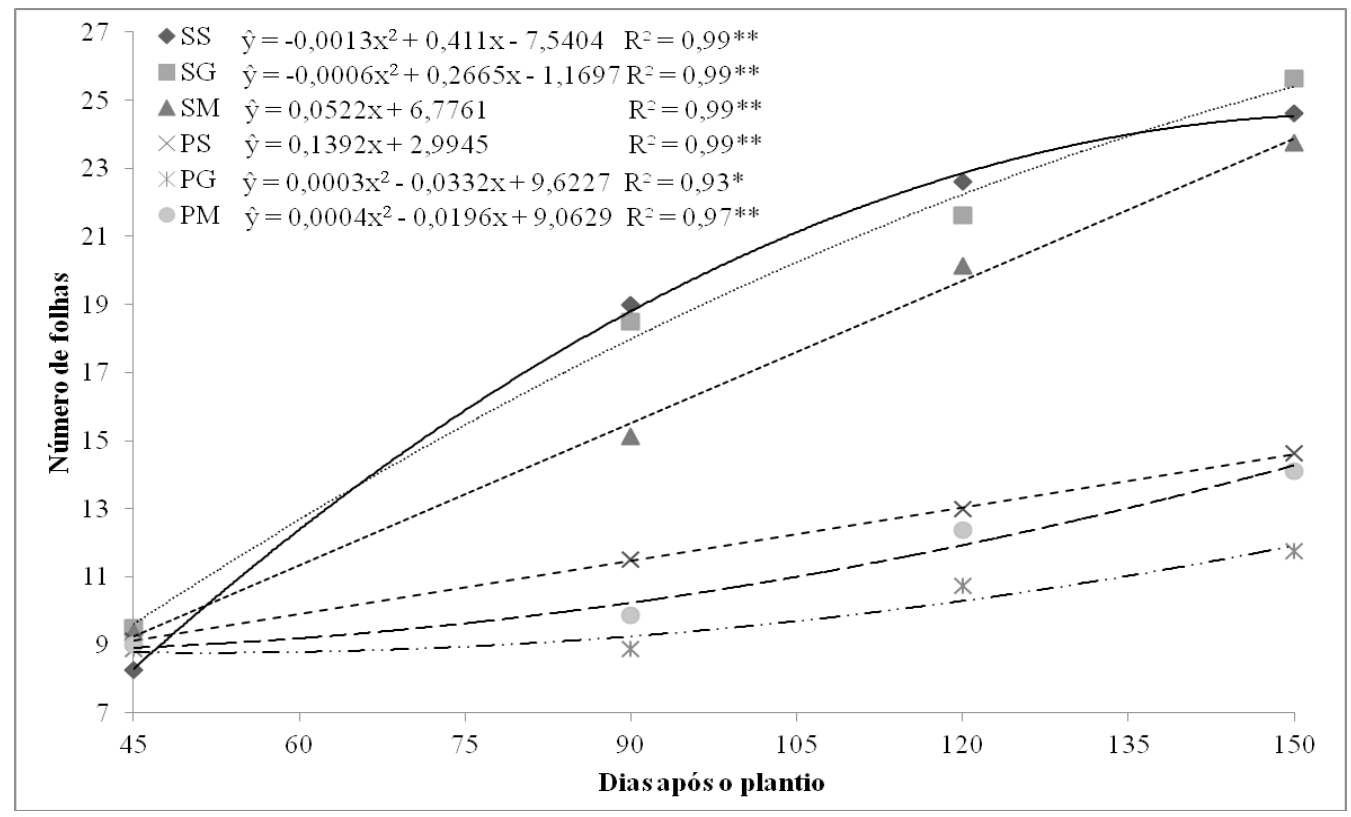

FIGURA 2 - Número de folhas em mudas do abacaxizeiro 'Pérola' e 'Smooth Cayenne', aplicado três tratamentos microbiológicos em quatro épocas de amostragem. Campos dos Goytacazes - Estado do Rio de Janeiro, 2009. * Significativo a $1 \% * *$ significativo a $5 \%$

Legenda: SS: 'Smooth Cayenne' não inoculado, SG: 'Smooth Cayenne' inoculado com G. etunicatum, SM: 'Smooth Cayenne' inoculado com mistura dos fungos Glomus clarum e Gigaspora margarita, PS: 'Pérola' não inoculado, PG: 'Pérola' inoculado com G. etunicatum, PM: 'Pérola' inoculado com mistura dos fungos Glomus clarum e Gigaspora margarita. 


\section{CONCLUSÕES}

1. A inoculação com FMAs no crescimento de rebentos oriundos de coroas de abacaxi não proporciona redução no tempo de formação das mudas.

2. Verificou-se, para a cultivar Pérola, que a utilização de FMAs reduziu o peso seco da parte aérea dos rebentos, e a utilização da mistura com duas espécies de fungos micorrízicos reduziu os teores de $\mathrm{P}$ e K.

3. A inoculação com FMAs no 'Smooth Cayenne' não promove incrementos no peso seco da parte aérea e no teor de K. No entanto, para o teor de P, foi verificado efeito benéfico da colonização micorrízica.

\section{REFERÊNCIAS}

ANJOS, E. C. T. dos; CAVALCANTE, U. M. T.; GONÇALVES, D. M. C.; PEDROSA, E. M. R.; SANTOS, V. F. dos; MAIA, L. C. Interactions between an arbuscular mycorrhizal fungus ( $\mathrm{Scu}$ tellospora heterogama) and the root-knot nematode (Meloidogyne incognita) on sweet passion fruit (Passiflora alata). Brazilian Archives of Biology and Technology Curitiba, v. 53, n. 4, 2010.

COELHO, R.I.; CARVALHO, A.J.C. de; LOPES, J.C.; TEIXEIRA, S.L.; MARINHO, C.S. Coroa do abacaxi 'Smooth Cayenne' na produção de mudas do tipo rebentão. Ciência e Agrotecnologia, Lavras, v.31, p.1867-1871, 2007.

FREITAS, M.S.M.; MARTINS, M.A.; CARVALHO, A.J.C. de. Crescimento e composição da menta em resposta à inoculação com fungos micorrízicos arbusculares e adubação fosfatada. Horticultura Brasileira, Brasília, n.24, p.11-16, 2006.

GRACE, C.; STRIBLEY, P. A afer procedure fot routine staining of vesicular-arbuscular mycorrhizal fungi. Mycological Research, Cambridge, v.95, p.1160-1162, 1991.

GUTIÉRREZ-OLIVA, V.F.; ABUD-ARCHILA, M.F.A.; ALVAREZ, S.J.D.; GUTIÉRREZ-MICELI, F.A. Influencia de los hongos micorrízicos arbusculares sobre el crecimiento de vitro plántulas de piña (Ananas comosus (L.) Merr.) Con diferentes niveles de fósforo. Gayana Botánica, Concepción, n.66, p.1-9, 2009.
JACKSON, M.L. Soil chemical analysis. New Jersey: Prentice Hall, 1965. 498 p.

JAIZME-VEGA, M.C.; AZCÓN, R. Effect of vesicular-arbuscular mycorrhizal fungi on pineapple [Ananas comosus (L.)Merr.]. Fruits, Paris, n.46, p.47-50, 1991.

LINS, G.M. de L.; TRINDADE, A.V.; ROCHA, H.S. Utilização de Gigaspora margarita em plantas micropropagadas de bananeira em diferentes estádios de enraizamento. Revista Brasileira de Fruticultura, Jaboticabal, v.25, n.1, p.143-147, 2003.

MALAVOLTA, E.; VITTI, G.C.; OLIVEIRA, S.A. Avaliação do estado nutricional das plantas: princípios e aplicações. 2. ed. Piracicaba: POTAFÓS, 319p. 1997.

MARSCHNER, H.; DELL, B. Nutrient uptake in mycorrhizal symbiosis. Plant and Soil, Dordrecht, n.159, p.89-102, 1994.

MATOS, A. P. de; REINHARDT, D. H.; SANCHES, N. F.; SOUZA, F. S.; TEIXEIRA, F. A.; ELIAS JÚNIOR, J.; GOMES, D. C. Produção de mudas sadias de abacaxi. Cruz das Almas: Embrapa Mandioca e Fruticultura Tropical, 2009. (Circular Técnica, 89).

SAMARÃO, S. S.; RODRIGUES, L. A.; MARTINS, M. A.; MANHÃES, T. N.; ALVIN, L. A. da M. Desempenho de mudas de gravioleira inoculadas com fungos micorrízicos arbusculares em solo não esterilizado, com diferentes doses de fósforo. Acta Scientiarum. Agronomy, Maringá, v. 33, n. 1, 2011.

SANTOS, P. C. dos. Produção de mudas do tipo rebentão, utilizando coroas de três variedades de abacaxi inoculadas com fungos micorrízicos 2009. 30 f. Monografia (Graduação em Agronomia) - Universidade Estadual do Norte Fluminense Darcy Ribeiro, Campos dos Goytacazes, 2009.

STANCATO, G. C.; SILVEIRA, A. P. D. da. Micorrização e adubação de mudas micropropagadas de antúrio, cv. Eidibel: crescimento e aclimatização ex vitro. Bragantia, Campinas, v. 69, n. 4, 2010. 
TRINDADE, A.V.; DANTAS, J.L.L.; ALMEIDA, F.P.; MAIA, I.C.S. Estimativa do coeficiente de determinação genotípica em mamoeiros (Carica papaya L.) inoculados com fungo micorrízico arbuscular. Revista Brasileira de Fruticultura, Jaboticabal, v.23, n.3, p.607-612, 2001.
WEBER, O.B.; SOUZA, C.C.M.; GONDIN, D.M.F.; OLIVEIRA, F.N.S.; CRISÓSTOMO, L.A.; CAPRON, A.L.; SAGGIN JÚNIOR, O. Inoculação de fungos micorrízicos arbusculares e adubação fosfatada em mudas de cajueiro-anão-precoce. Pesquisa Agropecuária Brasileira, Brasília, v.39, p.477-483, 2004. 